KARNIK, S. and PRABHU, R. 2021. Chemical detection of explosives in soil for locating buried landmines. In Bouma,

H., Prabhu, R., Stokes, R.J. and Yitzhaky, Y. (eds.) Counterterrorism, crime fighting, forensics and surveillance technologies V: proceedings of the 5th Counterterrorism, crime fighting, forensics, and surveillance technologies conference, part of the 2021 SPIE Security + defence conference, 13-17 September 2021, [virtual conference].

Proceedings of SPIE, 11869. Bellingham, WA: SPIE [online], article ID 118690A. Available from:

\title{
Chemical detection of explosives in soil for locating buried landmines.
}

\author{
KARNIK, S. and PRABHU, R.
}

(C) 2021 Society of Photo-Optical Instrumentation Engineers (SPIE). One print or electronic copy may be made for personal use only. Systematic reproduction and distribution, duplication of any material in this publication for a fee or for commercial purposes, and modification of the contents of the publication are prohibited. 


\title{
Chemical detection of explosives in soil for locating buried landmines
}

\author{
Shruti Karnik \& Radhakrishna Prabhu \\ School of Engineering, Robert Gordon University, Garthdee Road, Aberdeen, UK AB10 7GJ
}

\begin{abstract}
Trinitrotoluene (TNT) is a highly explosive nitroaromatic compound that is used for military and terrorist activities such as the development of improvised explosive devices (IEDs), landmines and is the main charge or explosive in most of the anti-personal and anti-vehicle mines. Different chemicals/ contaminants associated with TNT in soils near buried land mines comprise the microbial transformation products of TNT (2-amino-4,6-dinitrotoluene [2-Am-DNT] and 4-amino-2,6-dinitrotoluene [4-Am-DNT]), manufacturing impurities of TNT (2,4-DNT, 2,6DNT, and 1,3-DNB), and TNT. Time, cost, and casualties associated with demining have necessitated the demand for improved detection techniques with reduced false positives by directly detecting the explosive material, rather than casing material of mines. Different analytical methods used to detect trace level of explosives in soil include ion mobility mass spectrometry, gas chromatography-mass spectrometry (GC-MS), and liquid chromatographymass spectrometry (LC-MS) that require samples to be collected from hazardous sites to laboratories. This is extremely unsafe, time consuming, involve large and expensive instrumentation cost and specially trained staff. Thus, detecting chemical signatures of these nitroaromatics in soil infected with these chemicals due to leaked TNT mines can provide location of landmines/ landmine prone zones to aide humanitarian demining process. This paper illustrates soil analysis for explosives and selected contaminants by Raman spectroscopy as a chemical, nondestructive, remote sensing method. As with advancement of Raman-based standoff detection techniques, fieldportable instruments and UAV deployable probes, this technique can be effectively employed in detecting buried landmines based on specific chemical signatures of target analyte. In this present study, TNT-based nitroaromatic was assessed in contaminated soil samples using Raman spectroscopy, where uncontaminated soil was used as background and matrix for spiking target contaminants at different concentrations.
\end{abstract}

Keywords: Landmines, TNT, nitroaromatic explosives, Raman spectroscopy, remote sensing

\section{INTRODUCTION}

Landmines are weapons, usually buried, that explode when stepped on and are designed to injure or kill, leaving long-term psychological effects and posing a financial burden to the community. Landmines have an indefinite lifetime, and resulted in tremendous socio-economic disruptions for more than two decades ${ }^{1}$. Till this date, accurate records of the exact locations of the minefields are unmanaged, making removal of landmines painstakingly slow. Most affected are developing nations and underdeveloped nations, where the presence of landmines has hampered resettlement, agriculture, wildlife and tourism. Thus, the detection of explosives is a vital part of law enforcement, anti-terrorism, and military activities around the world. Deterioration of infrastructure and the threat posed by landmines restricted the ability of humanitarian organisations to travel and aid, due to the impact of mines on transport, loss of equipment and the safety of humanitarian staff. Other than the landmines sensitivity to explosion with time or atmospheric factors, absence of maps or information about the landmine types used or the areas where they were originally placed, the change of landmines locations due to climatic and physical factors, the large variety of types of antivehicle (AVM) and antipersonnel (APM) landmines, and the high cost of locating and removing landmines affect the process of humanitarian demining. The United Nations Department of Humanitarian Affairs (UNDHA) has very strict regulations in terms of humanitarian demining. As a matter of fact, the $99.6 \%$ of mines and unexploded ordnance must be safely removed from an area to consider it landminefree. Thus, there is growing demand for reliable landmine inspection systems in combination of an unmanned aerial system (UAV) applied for the terrain mapping and geo-detection of explosive landmine-like objects ${ }^{2}$. Drones, including unmanned air vehicles (UAVs) and micro air vehicles (MAVs), have been used for a variety of civilian and military applications and missions. These unmanned flying systems are able to carry different sensors based on the type of their missions, such as acoustic, visual, chemical, and biological sensors ${ }^{3}$. 
A typical landmine consists of different components- firing mechanism, detonator or igniter, booster charge, main charge, and casing. The booster charge is a highly sensitive explosive that will explode easily, when subjected to the shock of the detonator ${ }^{4}$. Typically, a pea-sized pellet of RDX is used. The purpose of the booster is to amplify the shock of the detonator and initiate the main explosive charge. The most common content of main charge mainly consists of loadings of few grams to Kilograms TNT, RDX, HMX explosives depending on the type of mine- i.e. antipersonnel mine (APM) or anti vehicle mine (AVM). The mine casing houses the components of the landmine and protects it from its environment and can

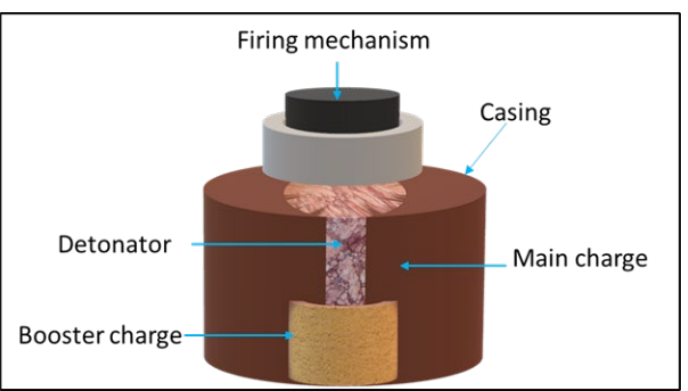

Figure 1 Composition of a landmine be metal, plastic, wooden etc. Detection of landmine in soil is affected by many different factors, including type of mine, type of explosive, type of casing and geographical features of the land, vegetation-such as type of soil, moisture content, topography etc. Detection of buried and partially buried landmines employ different techniques such as GPR, metal detectors, NIR and SWIR, microwave radar and visible light detection etc ${ }^{5-10}$. These techniques, however, suffer from many drawbacks and have resulted in significant false alarms, hence, detection of chemical signature of mine/ explosive is preferable to reduce these false alarms. Moreover, leakage and leaching of explosives from mine casing into soil, groundwater can occur due to wearing of casing and various environmental reasons including flood, landslides etc. Thus, detection of explosives and trace vapors in soil can be employed for detecting landmine affected zones/ areas. Since, TNT is commonly employed explosive in landmines, military devices and improvised explosive devices (IEDs), contamination of soil and groundwater by TNT based explosives and their degraded by-products has caused adverse effects on general population, vegetation and wildlife in these landmine prone areas and artillery bases ${ }^{11-13}$. Analytes most frequently found in soils collected near buried land mines were the microbial transformation products of TNT- (2-amino-4,6-dinitrotoluene [2-Am-DNT] and 4-amino-2,6-dinitrotoluene [4-Am-DNT]), manufacturing impurities of TNT (2,4-DNT, 2,6DNT, and 1,3-DNB), 2- Nitrotoluenes and TNT ${ }^{14,15}$. Different methods for characterizing hazardous waste sites are time-consuming, cumbersome, risky, and expensive ${ }^{16-19}$. Typically, site characterization requires a preliminary site assessment, followed by sampling of potentially contaminated soils and waters. The samples are sent to laboratories for analysis using EPA-certified methods.

In the present study, we have investigated the detection of 2-Nitrotoluene, an explosive and one of the degradation products of TNT, in soil and water samples using Raman spectroscopy. These samples can be treated as representative of explosive rich soil and groundwater systems. Raman spectroscopy is an excellent technique for detection of various chemical entities based on their molecular structure and each molecule has a unique chemical signature $^{20,21}$. The technique is very amenable to materials that reside in an aqueous environment (in contrast to infrared spectroscopy1); analysis is possible on solids, liquids, gases, and dissolved species. Different Raman techniques have been applied in detection of trace explosives in IEDs ${ }^{22-24}$. With the development of powerful laser sources and sensitive detectors, various portable and mobile Raman spectroscopy systems, standoff systems ${ }^{25}$, UAV drone fitted Raman surveillance sensors have increasing chances of being adopted in detection of explosives in landmines and sea-mines.

\section{Materials and Methods}

Materials:

Uncontaminated soils were used as background blanks and as the matrix for spiking target contaminant i.e. 2Nitrotoluene NT, that was purchased from Sigma Aldrich. Analytical grade Acetonitrile was used to prepare 2Nitrotoluene NT spiked soil samples. Raman measurement of 2-Nitrotoluene NT was recorded on a Raman system from Avalon (Raman Station R3)-benchtop Raman with $785 \mathrm{~nm}$ laser (300mW) and air-cooled CCD $\left(-50{ }^{\circ} \mathrm{C}\right)$ detector. The working distance for the probe was $5 \mathrm{~mm}$ and spectral range 250 to $3500 \mathrm{~cm}^{-1}$ at a resolution of $2 \mathrm{~cm}^{-}$ 1 .

Experimental method:

In this work, we have undertaken a preliminary investigation for exploring Raman spectroscopy as a feasible method for detecting nitroaromatics and explosives qualitatively under different conditions. The experiment was divided into three sets- dry soil sample spiked with NT, wet soil sample spiked with NT and water spiked with 
NT. These samples can be treated as tentative representative samples for different environmental sources contaminated with leaked mines/ explosive residue such as dry soil and ground, wet soil, and groundwater and water bodies such as lakes, ponds etc. For preparing dry soil samples, uncontaminated soil (100 mg) was suspended in $2 \mathrm{~mL}$ of acetonitrile and different amounts of 2-nitrotoluene NT was added under stirring to obtain 5 sets of samples S1-S5 with w/w ratio of soil: NT as 1:1, 1:0.8, 1:0.6,1:0.4 and 1:0.2. The mixture of soil and NT in acetonitrile was stirred, and solvent was allowed to evaporate at room temperature, dry samples were stored in desiccator for $24 \mathrm{~h}$ prior to Raman investigation. Similarly, for samples in wet soil, initially soil sample (100 mg) was added to $1 \mathrm{~mL}$ of deionised water and the sample was agitated in a sonication bath. NT was spiked in different amounts to the agitated soil sample such that the $\mathrm{w} / \mathrm{w}$ ratio of wet soil to NT was maintained as 1:1, 1:0.8, 1:0.6,1:0.4 and 1:0.2 in samples S1a, S2a, S3a, S4a and S5a. The sample was again agitated under sonication for 2 hours and was taken out only during the Raman analysis. For samples in water, NT was added to deionised water $(100 \mathrm{mg}) \mathrm{keeping}$ the $\mathrm{w} / \mathrm{w}$ ratio of water to NT as 1:1, 1:0.8, 1:0.6,1:0.4 and 1:0.2 in samples S1.1, S2.1, S3.1, S4.1 and S5.1. These samples were agitated in a sonication bath for 2 hours until each sample was procured for analysis and Raman spectrum was recorded for the milky emulsion sample type.

\section{RESULTS AND DISCUSSION}

Raman scattering for pure 2-Nitrotoluene NT were investigated (Figure 2) and sharp peaks for - $\mathrm{NO}_{2}$ group, $-\mathrm{CH}_{3}$ vibrations at 1612 (very weak vw), 1578 (medium m), 1520 (vw), 1344 (very strong vs), 1200 (weak w), 1162 $(\mathrm{w}), 1050(\mathrm{~m}), 858(\mathrm{w}), 792(\mathrm{~s}), 664(\mathrm{w})$ and $538(\mathrm{vw}) \mathrm{cm}-1$ were seen. Sharp peak at $1344 \mathrm{~cm}^{-1}$ corresponds to $\mathrm{NO}_{2}$ symmetric mode, two bands at $1578 \mathrm{~cm}^{-1}$ and $1520 \mathrm{~cm}^{-1}$ asymmetric $\mathrm{NO}_{2}$ stretching and aromatic $\mathrm{NO}_{2}$ conjugation, respectively. Raman bands at $1200 \mathrm{~cm}^{-1}$ and between 1162 to $1050 \mathrm{~cm}^{-1}$ corresponds to $\mathrm{H}-\mathrm{C}-\mathrm{C}$ in plane bending and methyl $\mathrm{H}-\mathrm{C}-\mathrm{H}$ asymmetric bend. Bands around $858 \mathrm{~cm}^{-1}$ and $792 \mathrm{~cm}^{-1}$ correspond to $-\mathrm{NO}_{2}$ and $-\mathrm{CH}_{3}$ vibrations, respectively.

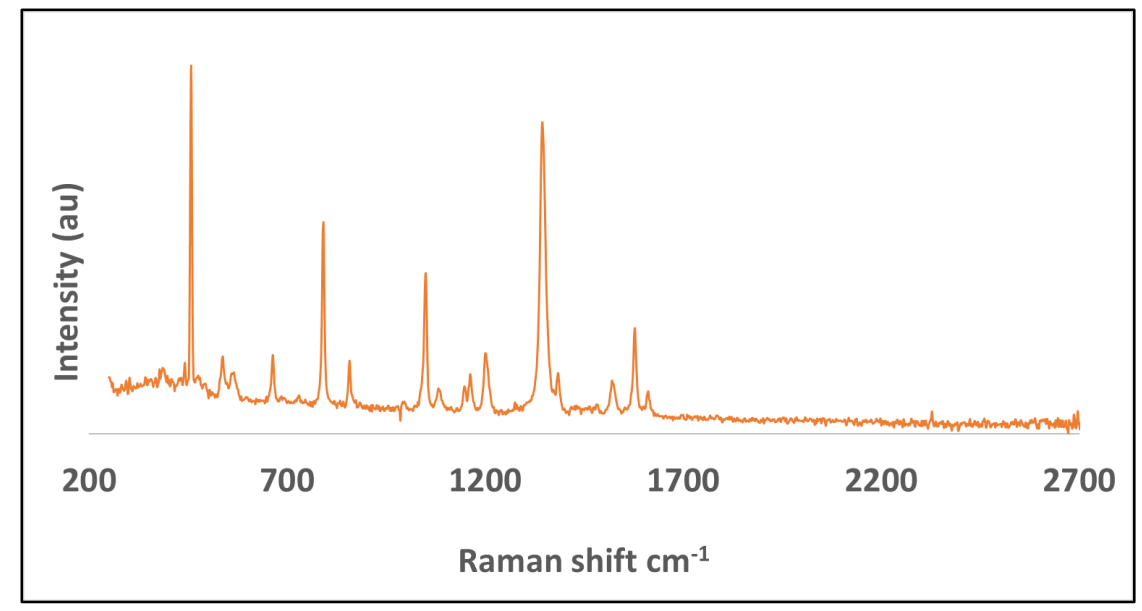

Figure 2 Raman spectrum of pure 2-Nitrotolunene NT

As seen in Figure 3, spiked NT in dry soil samples in different w/w ratio of soil: NT, S1- 1:0.2 w/w, S2- 1:0.4 $\mathrm{w} / \mathrm{w}, \mathrm{S} 3-1: 0.6 \mathrm{w} / \mathrm{w}, \mathrm{S} 4-1: 0.8 \mathrm{w} / \mathrm{w}$ and S5- 1:1 w/w, NT represents pure nitrotoluene and uncontaminated soil is blank or reference for soil. As seen in pure spectrum of NT, a sharp and a strong band at $1344 \mathrm{~cm}^{-1}$ for $-\mathrm{NO}_{2}$ group, can be seen as a very small signal in samples S3, S4 and S5. The sample acquisition time was dependant on the number of pulses and exposure time, in each of these cases, number of pulses were 10 and exposure time was 2Sec. This Raman band can be used to detect or identify the presence of nitroaromatics or TNT-based explosives qualitatively. Further optimisation of Raman spectroscope and signal enhancement with respect to different applications can be obtained to detect the nitroaromatics not just qualitatively but quantitatively as well in different environmental samples on-site. 


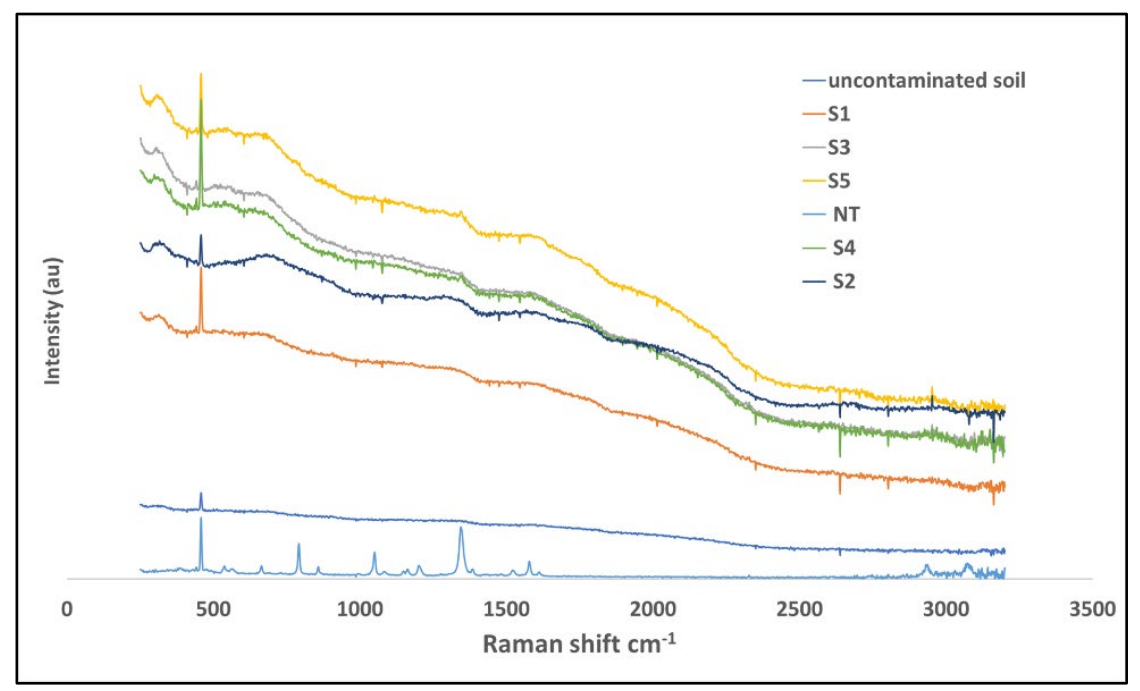

Figure 3 Raman spectrum of NT spiked dry soil samples

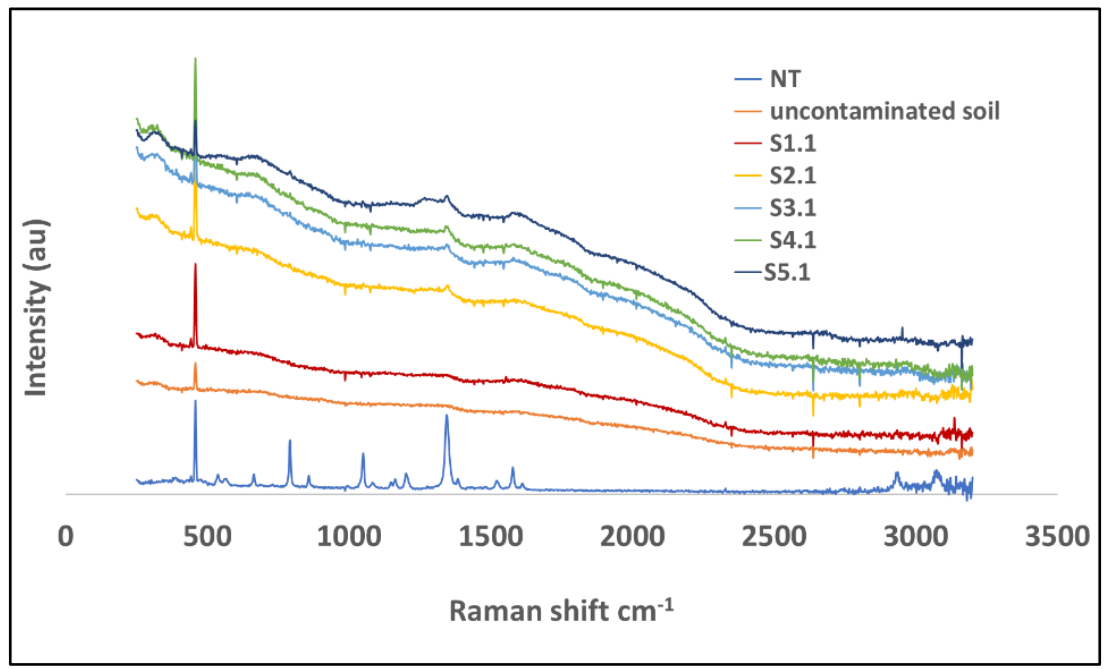

Figure 4 Raman spectra of NT spiked wet soil samples

Wet soil samples that are spiked with NT in different w/w ratios are denoted as S1.1, S2.1, S3.1, S4.1 and S5.1 and their Raman spectrum is represented in Figure 4. The number of pulses were 3 and exposure time was $2 \mathrm{Sec}$. It is obvious from the Figure 4, that the most prominent peak at $1344 \mathrm{~cm}^{-1}$ can be seen as a small signal in samples S3.1, S4.1 and S5.1. Another peak at $792 \mathrm{~cm}^{-1}$ was also observed in S5.1 which is the next strongest band seen in pure sample. These samples represent different environments or sites contaminated with explosives such as areas with silts, groundwater, swamps, moist soils or tropical regions and wetlands etc. Thus, further optimisation of Raman signal for specific application of detecting landmines or sea mines can be adapted in future. 


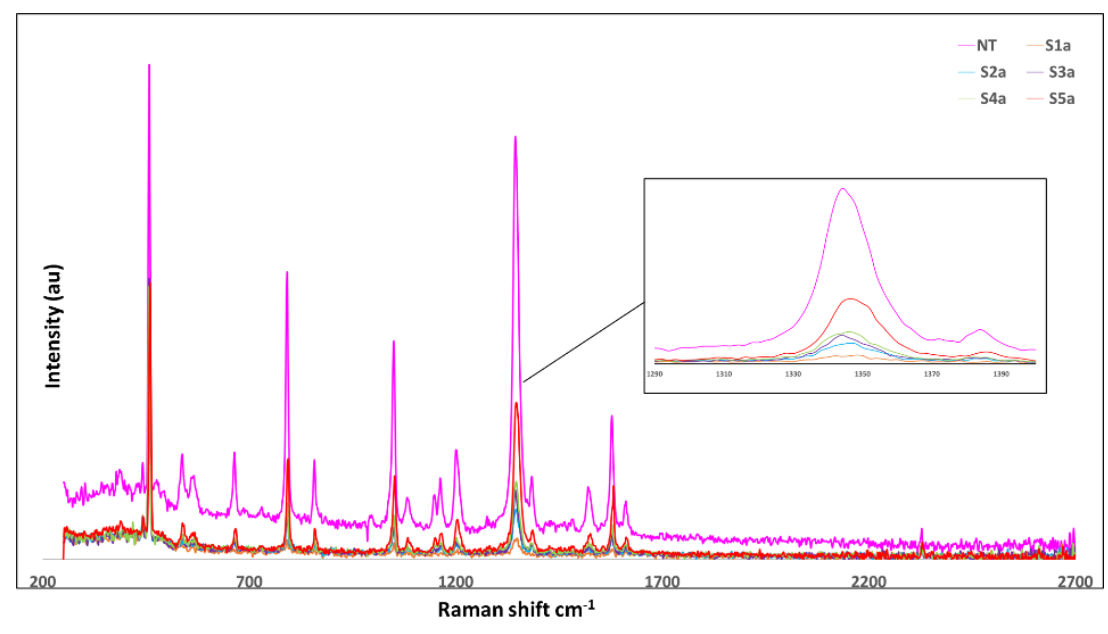

Figure 5 Raman spectrum of pure 2-Nitrotoluene NT

As depicted in Figure 5, where sample NT represents pure 2-Nitrotoluene whereas, sample S1a, S2a, S3a, S4a and S5a represent NT spiked water samples in different $\mathrm{w} / \mathrm{w}$ ratio of water: NT -1:0.2, 1:0:4, 1:0.6, 1:0.8 and 1:1 respectively. In each of these cases, number of pulses was 1 and exposure time was $2 \mathrm{Sec}$. Since water has a weak absorption in Raman between 3600 to $3700 \mathrm{~cm}^{-1}$, most of the medium to strong bands for Nitrotoluene were seen in all the samples. Almost all medium to strong bands can be seen notably in Raman spectrum of all the samples. Especially, the strong band at $1344 \mathrm{~cm}^{-1}$ was observed for the sample S1a with lowest concentration of Nitrotoluene. Thus, there is good scope of further optimisation and carrying out concentration dependant study. This investigation shows that Raman probe can be used in detecting explosives from landmines leaked into different waterbodies and water resources where other probes such as GPR, IR and other optical sensors fail.

\section{CONCLUSION}

This paper entails detection of nitroaromatics, TNT based explosive using Raman spectroscopy as a means of capturing chemical signature of landmines or leaked explosive to identify the landmine prone zones. NT are explosives and used as taggants in military grade TNT ammunitions as the trace vapours are easily detectable. NT is also found as a metabolite of microbial decomposition of TNT. Thus, detecting these chemicals in real environment/ landmine affected zones can give information of areas or soils infected with these chemicals due to leaked TNT mines irrespective of the casing type. In this work, three scenarios were investigated - Nitrotoluene contaminated dry soil, Nitrotoluene contaminated wet soil and Nitrotoluene contaminated water to mimic explosive rich natural environment such as dry lands, deserts, groundwater, wetlands, silts, river, and lakes etc. Strong Raman signal at $1344 \mathrm{~cm}^{-1}$ representing $-\mathrm{NO}_{2}$ group stretching can be seen in samples with all the three scenarios. Although, the signal was relatively much stronger in case of water rich samples than soil samples. Raman signal strength with respect to different samples was achieved in the following order: water contaminated samples $>$ wet soil samples $>$ dry soil samples. This must be due to the huge background generated from the soil and further optimisation and elimination of background can make this technique suitable in different environments. Raman scattering can further be complemented with LIDAR, LIBS or different optical methods for hybrid systems deployable on drones in landmine detection.

\section{REFERENCES}

[1] Duttine, A. and Hottentot, E., "Landmines and explosive remnants of war: a health threat not to be ignored.," Bull. World Health Organ., 91, 160-160A (2013).

[2] Colorado, J., Mondragon, I., Rodriguez, J. and Castiblanco, C., "Geo-Mapping and Visual Stitching to Support Landmine Detection Using a Low-Cost UAV,” Int. J. Adv. Robot. Syst. 12(9) (2015).

[3] Shahmoradi, J., Talebi, E., Roghanchi, P. and Hassanalian, M., "A comprehensive review of 
applications of drone technology in the mining industry," Drones 4(3), 1-25 (2020).

[4] Kasban, H., Zahran, O., Elaraby, S. M., El-Kordy, M. and Abd El-Samie, F. E., "A comparative study of landmine detection techniques," Sens. Imaging 11(3), 89-112 (2010).

[5] Lombardi, F., Griffiths, H. D., Lualdi, M. and Balleri, A., "Characterization of the Internal Structure of Landmines Using Ground-Penetrating Radar,” IEEE Geosci. Remote Sens. Lett. 18(2), 266-270 (2021).

[6] Ambrosanio, M., Bevacqua, M. T., Isernia, T. and Pascazio, V., "Performance Analysis of Tomographic Methods against Experimental Contactless Multistatic Ground Penetrating Radar," IEEE J. Sel. Top. Appl. Earth Obs. Remote Sens. 14, 1171-1183 (2021).

[7] Anna, L., Sten, N., Stefan, S. and Magnus, U., "Optical methods for detection of minefields." (2004).

[8] Zare, A., Bolton, J., Gader, P. and Schatten, M., "Vegetation mapping for landmine detection using long-wave hyperspectral imagery," IEEE Trans. Geosci. Remote Sens. 46(1), 172-178 (2008).

[9] Du Bosq, T. W., Lopez-Alonso, J. M., Boreman, G. D., Muh, D., Grantham, J. and Dillery, D., "Millimeter wave imaging system for the detection of nonmetallic objects," Detect. Remediat. Technol. Mines Minelike Targets XI 6217, 621723 (2006).

[10] Hamza, M., Jehangir, A., Ahmad, T., Sohail, A. and Naeem, M., "Design of surveillance drone with Xray camera, IR camera and metal detector," 2017 Ninth Int. Conf. Ubiquitous Futur. Networks, 111-114 (2017).

[11] Ostrinskaya, A., Kunz, R. R., Clark, M., Kingsborough, R. P., Ong, T. H. and Deneault, S., "Rapid Quantitative Analysis of Multiple Explosive Compound Classes on a Single Instrument via FlowInjection Analysis Tandem Mass Spectrometry," J. Forensic Sci. 64(1), 223-230 (2019).

[12] Psillakis, E., Naxakis, G. and Kalogerakis, N., "Detection of TNT-Contamination in spiked soil samples using SPME and GC/MS," Glob. NEST J. 2(3), 227-236 (2018).

[13] Jenkins, T. F., Leggett, D. C., Miyares, P. H., Walsh, M. E., Ranney, T. A., Cragin, J. H. and George, V., "Chemical signatures of TNT-filled land mines," Talanta 54(3), 501-513 (2001).

[14] Nepovim, A., Hebner, A., Soudek, P., Gerth, A., Thomas, H., Smrcek, S. and Vanek, T., "Degradation of 2,4,6-trinitrotoluene by selected helophytes," Chemosphere 60(10), 1454-1461 (2005).

[15] Esteve-nu, A. and Ramos, J. L., "Biological Degradation of 2 , 4 , 6-Trinitrotoluene," 335-352 (2001).

[16] Ezoe, R., Imasaka, T. and Imasaka, T., "Determination of triacetone triperoxide using ultraviolet femtosecond multiphoton ionization time-of-flight mass spectrometry," Anal. Chim. Acta 853(1), 508$513(2015)$.

[17] Alonso, D. E., Binkley, J., Kelly, C., Corporation, L. and Joseph, S., "Comprehensive Detection \& Confirmation of Explosive Residues in Soil Using a High Performance Benchtop GC-TOFMS," 49085.

[18] Walsh, M. E., "Determination of nitroaromatic, nitramine, and nitrate ester explosives in soil by gas chromatography and an electron capture detector," Talanta 54(3), 427-438 (2001).

[19] Milligan, K., Shand, N. C., Graham, D. and Faulds, K., "Detection of Multiple Nitroaromatic Explosives via Formation of a Janowsky Complex and SERS," Anal. Chem. 92(4), 3253-3261 (2020).

[20] Nachtmann, M., Deuerling, J. and Rädle, M., "Molecule sensitive optical imaging and monitoring techniques - A review of applications in micro-process engineering," Micromachines 11(4) (2020).

[21] Kiefer, W., "The Raman effect?a unified treatment of the theory of Raman scattering by molecules. Derek A. Long, John Wiley \& Sons, Ltd., 2002, Pp 597. ISBN 0-471-49028-8,” J. Raman Spectrosc. (2003).

[22] Zapata, F., López-López, M. and García-Ruiz, C., "Detection and identification of explosives by surface enhanced Raman scattering,” Appl. Spectrosc. Rev. 51(3), 207-242 (2016).

[23] Tuschel, D. D., Mikhonin, A. V., Lemoff, B. E. and Asher, S. A., "Deep ultraviolet resonance raman excitation enables explosives detection," Appl. Spectrosc. 64(4), 425-432 (2010).

[24] Johny, J., Karnik, S. and Prabhu, R., "Investigations of hollow-core photonic crystal fibres (HC-PCF) for trace explosive vapour detection," 23 (2020).

[25] Zachhuber, B., Ramer, G., Hobro, A., Chrysostom, E. T. H. and Lendl, B., "Stand-off Raman spectroscopy: A powerful technique for qualitative and quantitative analysis of inorganic and organic compounds including explosives," Anal. Bioanal. Chem. 400(8), 2439-2447 (2011). 